\title{
¿Hubo ruptura epistemológica en la ciencia del siglo XIV?
}

\author{
Joan Rovira \\ Elena Carbonell
}

\section{Resumen}

El concepto de ruptura epistemológica como medida para saber si hay o no revolución científica en el siglo XIV. Posiciones enfrentadas sobre la posibilidad de una revolución epistemológica en física y en cosmología. El Ars Medica de Arnau de Vilanova como síntesis de la teología cristiana y el naturalismo griego. Conclusiones sobre el hombre medieval y su conocimiento del mundo.

Palabras clave: Aristóteles, Arnau de Vilanova, Galeno, Ptolomeo, cosmología, escolástica, física, método experimental, medicina, teología, «ruptura epistemológica».

\section{Abstract}

Epistemological rupture as a way to analyze if there was or not scientific revolution during the XIV ${ }^{\text {th }}$ century. The many different opinions on the possibility of epistemologic rupture in Phisics and Cosmology. The Ars Medica of Arnaldus de Vilanova as a synthesis of christian theology and greek naturalism. Conclusions about the medieval man and his knowledge of the world.

Key words: Aristotles, Arnaldus from Vilanova, Galenus, Ptolomeus, cosmology, scholastics, physics, experimental method, theology, epistemological rupture.

En Las memorias de Adriano, Marguerite Yourcenar nos habla de un mundo en el que los dioses paganos ya no eran creíbles y el Dios cristiano todavía no había llegado. La historia de Occidente nos sugiere, a veces, situaciones semejantes. Una de ellas es la que se produce en la ciencia de los siglos XIII y XIV, durante los cuales la autoridad aristotélica, aunque comúnmente aceptada, empezaba a ser cuestionada, y se tambaleaba precediendo un orden cosmológico y científico todavía por nacer. Para estudiar la distancia entre ambos estados de ciencia en el período que abordamos - la ciencia en el tiempo de Petrarca - vamos a utilizar el concepto de «ruptura epistemo- 
lógica». ${ }^{1}$ Este concepto, acuñado por G. Bachelard, intenta mostrar cómo la ciencia contemporánea - fundamentalmente a partir de la relatividad y la mecánica cuántica- rompe con las estructuras del sentido común de la ciencia precedente, desmarcándose de estas sin posible reconciliación. «Ruptura epistemológica» significa, por tanto, la adopción de un método o de una teoría nuevos que imposibilitan al conocimiento el retorno a las estructuras del saber anterior. En el presente discurso la pregunta que nos formulamos es si podemos aplicar el concepto de ruptura epistemológica a la situación de la ciencia del siglo XIV y en qué puntos, si los hay, ésta es capaz de prefigurar la revolución científica que conduciría al nacimiento y a la consolidación de un modelo positivista de ciencia durante los siglos posteriores.

Un aspecto a considerar como premisa necesaria a tal análisis es la perspectiva desde la cual se aborda la «objetividad» en la construcción de la historia de la ciencia. En algunas ocasiones, la huella del positivismo se traduce en un tratamiento reduccionista de la historia de la ciencia que, sin recurrir a métodos históricos o filosóficos, mide el valor de ésta únicamente por su capacidad de aportar conceptos, datos y experiencias que conducen a un estado de ciencia más avanzado. ${ }^{2}$ En otras interpretaciones, en cambio, la ciencia es interrogada por sus historiadores con el fin de hallar justificaciones extracientíficas que sirvan para sostener intereses y doctrinas determinados: es el caso de Pierre Duhem, ${ }^{3}$ que busca en el cristianismo medieval la incipiente conformación de una ciencia moderna; o de las interpretaciones marxistas que inscriben su curso dentro de las lecturas socioeconómicas que la direccionan. En cualquier caso, la historia de la ciencia tiene un carácter peculiar: no es una historia acumulativa, ni progresa linealmente, sino que se ofrece surcada de crisis y rupturas que infligen, mediante revoluciones científicas, la alternancia de paradigmas y de las cosmovisiones en ellos implícitas. ${ }^{4}$ Pues-

1. Gastón BACHELARD, Le rationalisme appliqué, París: PUF, 1949. La explicación del concepto de «ruptura epistemológica» se encuentra en el capítulo VI: "Connaissance commune et connaisance scientifique», p. 102-105.

2. “Cómo se hace la historia de la ciencia y cómo debería hacerse?», «De qué se hace la historia en historia de la ciencia?» Estas consideraciones han aparecido en ciertos debates sobre lo que los autores anglosajones designan con el nombre de «internalismo» y "externalismo». El externalismo es una forma de escribir la historia de las ciencias condicionando un cierto número de acontecimientos por sus relaciones con intereses económicos y sociales, con exigencias prácticas y técnicas, con las ideologías religiosas o políticas, etc. Por su parte, el internalismo - al que los externalistas consideran una forma de idealismo- aboga por la idea según la cual no hay historia de la ciencia posible si uno no se sitúa en el interior mismo de la obra científica. Cfr. Georges CANGUILHEM, «L'object de l'histoire des sciences» en ID. Études d'histoire et de philosophie des sciences, París: Vrin, 1970, p. 14-15.

3. Pierre DuHEM (1861-1916). Pionero del estudio de la ciencia medieval, autor entre otras obras de Le système du monde, París: 1913-1959, 10 vols. Duhem ha subrayado la continuidad en el desarrollo de la física desde la Edad Media hasta los tiempos modernos. Cfr. el artículo de Alexander KOYRÉ «Galilée et Platon» en ID. Études d'histoire de la pensée scientifique, París: PUF, 1973, p. 171-172.

4. Cfr., por ejemplo, Thomas KUHN, The structure of scientific revolutions, Chicago: The University of Chicago Press, 1965 
to que toda historia de la ciencia es inevitablemente juzgada desde un estado de la ciencia posterior, parece pertinente preguntarse, retrospectivamente, por la posibilidad de ruptura epistemológica en la ciencia bajomedieval del siglo XIV. Esbozaremos nuestra aproximación al tema a partir de tres ámbitos sucesivamente encadenados en un nivel epistemológico: la cosmología, la física y la medicina.

\section{La cosmología}

La gran filosofía medieval, surgida de la confluencia de tradiciones bizantina, hebrea y árabe gestada en universidades como la de París, Bolonia y Oxford forjó durante la Baja Edad Media una cultura latino-cristiana unitaria y vertebrada por el método escolástico. ${ }^{5}$ Si bien es cierto que en un primer momento la irrupción del aristotelismo en las universidades planteó el problema entre fe y razón —el estudio directo de la realidad del aristotelismo fue repetidamente condenado-, el tomismo bajomedieval supo aunar tales divergencias mediante una hábil teologización de la herencia clásica. Afirmando el papel de intervención de Dios en la función creadora, desmintiendo la negación aristotélica del infinito y la eternidad de la materia (que habría excluido el fin del mundo o la posibilidad de creación ex-nibilo), la filosofía bajomedieval pudo acoger las enseñanzas aristotélicas en la base de su caudal de saberes. De esta forma la asimilación medieval del aristotelismo sirvió, en parte, para emancipar el conocimiento de la consideración unilateral de los problemas teológicos (Duhem) ${ }^{6}$ orientándolo, al menos en el propósito, hacia el estudio de problemas concernientes al mundo físico. Por otra parte la recepción medieval de Aristóteles significó, sin embargo, la cristalización de la herencia clásica en principios de autoridad que - a pesar de ser divulgados y prolongados en incesantes discusiones escolásticas (realistas, nominalistas, etc.) — jamás llegaron a cuajar en la creación de un cuerpo científico enteramente renovado. Durante los siglos XIII y XIV cabría contraponer a la contumacia filosófica la indigencia científica: desde Alberto Magno hasta Nicolás de Oresme el saber medieval alcanzó sus más elevadas cotas, pero el humanismo helenizante había ido substituyendo progresivamente las influencias pseudo-árabes sin que la

5. Paolo Rossi, El nacimiento de la ciencia moderna en Europa, Barcelona: Crítica, 1998, p. $14-15$.

6. Tres razones se imponen en la Alta Edad Media a la idea de investigación científica que defendemos: 1) comprender que lo real no tiene ningún sentido para el hombre de la época, 2) la idea de progreso implícita en toda investigación no es comprensible, puesto que toda verdad accesible al hombre ha sido revelada a éste de una vez por todas en la Biblia, único origen del conocimiento, 3) la relación de causalidad, base necesaria de toda ciencia, es inconcebible. Con la escuela de Chartres se produce un verdadero renacimiento intelectual de Europa, es a través de ella que se difunde la idea según la cual si bien es cierto que Dios ha creado la naturaleza, también lo es que ésta ha sido dotada de leyes que Él respeta. Según esta escuela Dios es la causa primera de todo, pero existe un orden autónomo de causas segundas — las leyes - que se pueden estudiar racionalmente. Cfr. Jean-Paul LEVY, Le pouvoir de guerir, París: Ed. Odile Jacob, 1991, p. 133-134. 
ciencia obtuviera inmediato beneficio de ello ${ }^{7}$. Entre la autoridad de las fuentes clásicas asimiladas y el diálogo experimental con la realidad, el siglo XIV se decantó invariablemente por los principios de autoridad, sin interpelar la naturaleza con nuevas preguntas. Algunas figuras como Aristóteles o Galeno se erigieron como fortalezas inexpugnables, y aunque en ellas llegaran a surgir, ciertamente, algunas fisuras, no bastaba con hacer críticas de detalle para elaborar una nueva ciencia: había que demoler todo el edificio. ${ }^{8}$

En cosmología, la autoridad aristotélica se vio contestada por la teoría de los epiciclos y de las excéntricas de Ptolomeo, que se impuso durante la época tardomedieval al modelo de Eudoxo, el gran propagandista de la cosmología aristotélica. De esta derrota parcial del aristotelismo ¿supo sacar partido la ciencia medieval? El fracaso de la teoría aristotélica en un punto particular no comportó de ningún modo, en este caso, la destrucción de la coherencia del sistema: ciertamente, la astronomía de Ptolomeo podía medir mejor el espacio del cielo, pero no era capaz de explicar mejor el mundo; podía explicar cómo funciona el mundo, pero no cómo era éste. Como es bien sabido, habría que esperar hasta la revolución copernicana para que la imagen del mundo aristotélica - y también la de Ptolomeo- llegaran a desvanecerse; y hasta Kepler y Galileo para que el cosmos se transformara en universo, el mundo dejara de ser cerrado, y la materia se volviera homogénea y regida por una sola ley: la gravitación newtoniana. La ciencia moderna conllevaría, como primer imperativo, una nueva medida cuantitativa - y no cualitativa- de los fenómenos en su empeño de examinar el mundo como un poderoso autómata, y el hombre, como máquina. De esta forma la revolución científica moderna dibuja una cosmovisión radicalmente contrapuesta a la de los científicos tardomedievales: éstos, al no desmarcarse de la concepción teológica imperante en los sistemas escolásticos, no llegaron tampoco a desterrar definitivamente la filosofía aristotélica como núcleo de su pensamiento.

En definitiva nos cuestionamos, pues, ¿por qué no se desarrolló una crítica medieval capaz de poner en entredicho el pensamiento que daba soporte a este orden de las cosas? Seguramente la respuesta a este interrogante no viene dada por criterios que rigen la ciencia aisladamente, sino que depende en gran medida de factores presentes en el medio socio-político y cultural, el cual no propiciaba tales aventuras de la razón. Esto nos hace pensar que para que una nueva física tuviera ocasión de desarrollarse fueron precisos un conjunto de acontecimientos políticos —el individualismo de una sociedad renacentista, el libre albedrío, la disposición de interrogar numéricamente la naturaleza-

7. Cfr. Guy Beaujouan, «Visión sinóptica de la ciencia medieval en occidente», en Pedro LaÍN Entralgo (dir.) Historia Universal de la Medicina, vol. III, Barcelona: Salvat, 1972, p. 158.

8. «Ptolomeo, Aristóteles y Galeno dominaban como gigantes extraordinarios cuyas obras se alzaban a modo de formidables fortalezas. El hombre de la Edad Media abrió algunas brechas en las murallas, pero quedó como encerrado en el interior. El porvenir iba a mostrar que era ilusorio hacer críticas de detalle en lugar de proceder a la demolición total. Se imponía una revolución: la de Copérnico contra Ptolomeo, la de Galileo frente a Aristóteles, la de Harvey contra Galeno. Cfr. Guy Beaujouan, op. cit., p. 163. 
capaces de imponer la ruptura con el antiguo orden prevaleciente. Probablemente, Aristóteles fue desbancado antes por el curso de la política y de la economía - pensamos en la renovación latente en El príncipe de Maquiavelo, en el materialismo burgués en ascenso- que por la cosmología y la filosofía. No es casual que la nueva ciencia moderna, en su reivindicación de la importancia del cálculo, busque en Platón —y no en Aristóteles - su principio clásico de autoridad: la matematización total del universo desarrollada por Galileo y Newton se sostiene sobre la necesidad del número, el cual debería ir despojándose, progresivamente, de su inicial misticismo.

Podríamos preguntarnos, asimismo, por qué Platón, a diferencia de Aristóteles, no intervino en la construcción de la ciencia medieval cuando de hecho, el mayor de los aristotélicos, Santo Tomás, y el mayor de los platónicos, San Buenaventura, fueron exactamente contemporáneos. Ciertamente, la Edad Media conocía Platón, pero lo conocía de segunda mano (es el caso del Menón y el Fedón, que resultaron en general bastante desconocidos). Una suerte diversa fue la del Timeo, muy divulgado a través de la traducción de Calcídio, aunque se trataba de un texto de naturaleza ciertamente peculiar: el Timeo no es un libro de astronomía sino que más bien la historia del alma del mundo, una curiosa mezcla de teología y de física matemática, de cosmología mítica y de mecánica celeste. ${ }^{9}$ Su latente misticismo pudo ser recogido por astrólogos y alquimistas medievales, pudo también ser objeto de las más diversas supersticiones, pero su distancia para con el mundo real difícilmente podía encauzar la actividad científica bajomedieval: ésta, sin ser positivista, sí requería cierta capacidad de intervención activa sobre el mundo, una exigencia de la que la ciencia antigua, más contemplativa que operativa, había carecido. Con el Aristóteles entendido al modo bajomedieval existía un cierto espacio para la experiencia; por el contrario, la teoría del alma platónica en su interpretación medieval obstaculizaba cualquier hipotética proximidad con los objetos del mundo sensible. Koyré expone la visión medieval del platonismo sosteniendo que no es estudiando las cosas — los objetos del mundo sensible- como el alma conoce la verdad, puesto que la verdad de las cosas sensible no está en éstas, sino en su conformidad con las esencias eternas, con las ideas eternas de Dios. Son estas ideas, ideas como número o perfección, las que constituyen el objeto del saber verdadero, de modo que el pensamiento ha de llevarse hacia estas, apartándose del mundo dado a nuestros sentidos. ${ }^{10}$

Tal concepción se dibujaba demasiado distante a cualquier tipo de aspiración empírica, motivo por el cual durante el siglo XIV prevalecieron los esquemas aristotélicos sobre los platónicos. Lo hicieron, sin embargo, permaneciendo escasamente alterados, cristalizando, tras su asimilación con el cristianismo, en un conjunto de preceptos escolásticos que cosían el pensamiento a la tradición y lo mantenían cohesionado y unívoco. Como veremos al hablar de la

9. Cfr. Alexander KOYRÉ, "Aristotelicisme et platonisme», en Études d'histoire de la pensée scientifique, cit. p. 31 y s.

10. Alexander KOYRÉ, op. cit., p. 37. 
física (que a diferencia de la cosmología, perfila el espacio de posibilidad de la experiencia), el relativo empirismo de la actividad científica bajomedieval no fue suficiente para garantizar la eclosión de una ciencia propiamente experimental, capaz de conjuntar la práctica con la teoría y con la razón (propiciando la modificación de ésta, en el caso necesario).

\section{La física}

Algunos autores como Crombie ${ }^{11}$ han pretendido ver en la ciencia medieval el esbozo de un método experimental en presunta contradicción con la intuición aristotélica, limitada a la observación y de una naturaleza más bien contemplativa que operativa. Crombie postula que la teoría sistemática de la ciencia experimental era ya aplicada por un gran número de filósofos bajomedievales para poder producir una revolución técnica y metodológica. La mejora técnica durante el período que nos ocupa resulta efectivamente innegable: la construcción de innumerables iglesias, catedrales y conventos, los molinos de viento, las nuevas técnicas de arado pesado o la invención del estribo fueron, sin duda alguna, las grandes conquistas técnicas que sobrevinieron a una etapa de crecimiento demográfico y económico. También la técnica militar se transformó ostensiblemente durante la Guerra de los Cien años, en el espacio que mediaba entre dos batallas como Crecy (1346) y Azincourt (1415). Sin embargo, ¿debemos creer por ello que existe una ciencia experimental en la Edad Media y que ésta se opone a la ciencia teórica de los griegos? El progreso tecnológico no siempre implica un progreso científico: la unión indisociable entre ciencia y técnica es un hecho contemporáneo que se produce solamente durante el siglo XX, pero no antes. En ciertas sociedades el florecimiento de la tecnología ha podido producirse al margen del progreso científico, del mismo modo que el avance científico no necesariamente ha cabalgado siempre sobre un progreso tecnológico (pensemos en la civilización griega, que elaboró una ciencia de intenciones basada en la observación, pero no una ciencia de hechos, fundamentada en la experimentación). Por otra parte, el empirismo medieval, aunque ampliara las concesiones a la experiencia en relación con la ciencia antigua, permanecía aferrado a una concepción inmovilista del saber: el recurso a la experiencia no servía a una modificación de la razón ni involucraba necesariamente el ámbito de las ideas, el cual se ofrecía más bien enturbiado por los obstáculos epistemológicos. ${ }^{12}$ Es significativo el hecho de que la «teoría del

11. August C. Crombie, Robert Grosseteste and the origins of experimental science. Agustin to Galileo, Oxford: Clarendon Press, 1959.

12. «Hay que plantear el problema del conocimiento científico en términos de obstáculos... En el mismo acto de conocer, aparecen íntimamente, por una especie de necesidad funcional, pausas e inquietudes... Lo real no es nunca "lo que podríamos pensar", sino lo que hubiéramos debido pensar... La opinión está siempre, por derecho propio, en un error. La opinión piensa mal; no piensa: traduce las necesidades en conocimientos. Al designar a los objetos por su utilidad, se niega a conocerlos. No se puede basar nada sobre la opinión: antes hay que destruirla. Es el primer obstáculo que hay que superar. El espíritu científico nos prohi- 
impetus» de J. Buridan, que desmentía la física aristotélica en lo relativo al movimiento de los proyectiles apelando a la experiencia común, no repercutiera en una crítica más extensa, ni implicara la revisión o el abandono de las teorías aristotélicas.

Aristóteles había afirmado que el movimiento de un proyectil abandonado a sí mismo era debido al desplazamiento del aire. Buridan, contemporáneo de Petrarca, intentó rebatir tal razonamiento sosteniendo que en caso de que así fuera, las briznas de paja transportadas por un carro en movimiento deberían caer hacia delante y no hacia atrás. Ante la contradicción entre la autoridad y los hechos, la balanza se inclinó nuevamente a favor de Aristóteles, autor no solamente de una física sino de toda una cosmología capaz de abrazar el mundo y de proporcionar explicaciones cerradas y probablemente más conciliadoras con las expectativas coetáneas. El saber escolástico no era todavía un saber dispuesto a interrogar directamente a la naturaleza, sino más bien un saber que se interrogaba a sí mismo, proporcionándose explicaciones del ámbito maestrodiscípulo (pero no del inventor). ${ }^{13}$ El empirismo medieval puede entenderse, de este modo, como contrapuesto a la experimentación moderna, especialmente concebida para "forzar a la naturaleza a revelar sus secretos».

En lo que a la metodología se refiere, los filósofos medievales continuaron las discusiones que sostuvieron los griegos, sobre todo Aristóteles - es el caso de los Segundos analíticos - en relación con el dualismo procedimental inducción-deducción. En el siglo XIII, Roberto Grossatesta fue el primero en reconocer los dos problemas metodológicos de la «verificación» y la «falsación» experimentales que se plantearon cuando la concepción griega de la demostración geométrica fue aplicada al mundo de la experiencia. Sin embargo, la extendida consciencia de los métodos de comprobación y falsación a lo largo de los siglos XIII y XIV no constituye garantía suficiente para postular la articulación de una ciencia experimental: la ciencia experimental exige cálculo, y la aplicación sistemática del cálculo no constituiría propiamente una exigencia hasta que la revolución científica forzara la transición de un mundo cualitativo al mundo cuantitativo.

A diferencia de Crombie, ${ }^{14}$ que sitúa en la revolución técnica y metodológica de los siglos XIII y XIV el nacimiento a una nueva ciencia y el factor deter-

be tener una opinión sobre temas que no comprendemos, sobre preguntas que no sabemos formular claramente. Ante todo hay que saber plantear los problemas. Y a pesar de lo que se diga, en la vida científica los problemas no se plantean por sí mismos. Precisamente este sentido del problema da el carácter de verdadero espíritu científico. Para un espíritu científico cualquier conocimiento es una respuesta a un pregunta. Si no ha habido pregunta no puede haber espíritu científico. Nada es evidente. Nada es dado. Todo se construye», en Gaston BACHELARD, La formation de l'esprit scientifique, Paris: Vrin, 1938, p. 13-14.

13. Paolo Rossi, El nacimiento de la ciencia moderna en Europa, Barcelona: Crítica, p. 15-16.

14. August C. Crombie, Robert Grosseteste..., cit. «El rasgo distintivo del método científico del siglo XVII, si se lo compara con el de la antigua Grecia, era su concepción de la forma en que una teoría debía relacionarse con los hechos observados que se proponía explicar, la serie de pasos lógicos que comportaba para edificar teorías y someterlas a los controles experimentales. La ciencia moderna debe su éxito, en buena parte, al uso de estos métodos 
minante para su progreso, creemos más bien que una metodología no puede constituir por sí sola una ciencia sin peligro de reduccionismos. La ciencia es otra cosa, y la revolución científica apunta necesariamente hacia el ámbito más lejano de las estructuras del conocimiento: es significativo que los máximos artífices de ésta - Copérnico, Vesalio, Galileo-dialogaran directamente, en el plano teórico, con los científicos de la antigüedad -Aristóteles, Platón, Galeno- y no con los filósofos medievales. En definitiva, pues, para el nacimiento de la ciencia moderna habrían de superarse las limitaciones del empirismo aristotélico, implantando un método experimental que concediese la predominancia a la razón sobre la simple experiencia y sustituyera esta realidad empírica por los modelos matemáticos. Esto significa la primacía de la teoría sobre los hechos, y por tanto, la superación del empirismo ingenuo por un racionalismo que alcanzaría su máxima expresión en la filosofía de Descartes.

\section{La medicina: anatomía, ars medica}

Durante la Baja Edad Media, con la irrupción de la escolástica en las universidades, el centro de gravedad del conocimiento médico se desplazó de la escuela de Salerno - mito fundacional de la ciencia medieval - a Boloña, ciudad en la que se desarrolló un importante renacimiento de anatomía encabezado por Mondino de Luzzi. Tras realizar las observaciones pertinentes, Mondino de Luzzi propició una revolución de la nomenclatura anatómica dotando esta disciplina de un nuevo léxico en el que los términos árabes usados en la descripción del cuerpo humano eran substituidos por términos griegos. Las contribuciones de Mondino a la disciplina anatómica fueron sin duda notables: recordemos que ésta, a diferencia de la física, es una disciplina de observación y no de cálculo - Vesalio fue un observador; Copérnico, un calculador-. ${ }^{15}$ A pesar de ello, la trascendencia de las aportaciones de Mondino quedó minimizada a causa de su ausencia de continuadores, no pudiendo así cristalizar en una ciencia anatómica capaz de desmarcarse de las fuentes clásicas de las cuales la medicina medieval bebía. Lo mismo ocurría, simultáneamente, en la escuela Montpellier, donde el desarrollo de la cirugía de Guy de Chauliac, al no alcanzar ninguna continuidad, acabó aplazando la revolución científica hasta Ambroise Paré. Volviendo a Mondino de Luzzi, observamos como las líneas esenciales de su pensamiento anatómico permanecen ancladas al galenismo (por ejemplo, en lo concerniente a su doctrina de las tres cavidades y los tres espíritus). En un plano más general, las resonancias

inductivos y experimentales que constituyen lo que se llama a menudo "método experimental”». El libro de Crombie sostiene la tesis de que la comprensión sistemática moderna, por lo menos en los aspectos cualitativos de este método, es debida a los filósofos del siglo XIII: «ellos son los que han transformado el método experimental de los griegos y han empezado la ciencia experimental moderna", en Études d'histoire de la pensée scientifique, cit., p. 62.

15. Georges Canguilhem, «L'homme de Vesale dans le monde de Copernic», en Études d'histoire et de philosophie des sciences, cit., p. 28. 
filosóficas - Galeno, el Juramento Hipocrático, Aristóteles, Avicenna o Averroes- o teológicas parecen prolongar sus ecos en cada concepto, en cada obra y en cada tratado de la medicina medieval. ${ }^{16}$

Las definiciones medievales de la salud fueron, sustancialmente, aplicaciones y reformulaciones de las numerosas fuentes clásicas y coetáneas. La recepción del pensamiento de Aristóteles, encauzada por medio de las fuentes árabes y del averroísmo, fue inicialmente causa de controversia, pero con el tiempo, y sobretodo a partir de Alberto Magno y Tomás de Aquino, llegaría a ser concebido y asimilado como único pensamiento verdaderamente cristiano. Los escolásticos heredaron de Aristóteles la noción de techné o ars entendida como «recta razón", como técnica o "saber hacer» médico en el que se manifestaba la fronesis o prudencia. El dogma médico central era que la enfermedad se producía por causa de un desequilibrio (por exceso o por defecto), de modo que la medicina debía actuar para reequilibrar el desorden, indagando a fin de encontrar la correcta adecuación de cada substancia en cada paciente. También en las universidades emergentes se difundía y se transmitía este tipo de conocimiento de tipo racional y fideísta a la vez. Arnau de Vilanova, por ejemplo, creó un método basado en el empirismo rudimentario medieval (sin llegar a formular un método experimental), pero que debía ser dirigido por la razón, por la luz natural y, por tanto, por los atributos del sentido común (sineresis) propios del alma. ${ }^{1}$

Este acercamiento de la medicina a la práctica o «dirigismo» supone, quizás, la consecuencia más palpable de la revolución teológica que separa Aristóteles y Arnau de Vilanova, la cual conduciría hacia lo que Zubiri ha llamado «la introducción, en filosofía, de una nueva idea del mundo». ${ }^{18}$ En la revolución aludida, la medicina bajomedieval fusionaba las enseñanzas aristotélicas con la introducción de nuevos conceptos teológicos tales como el concepto de "potencia ordenadora de Dios», la noción de "causa segunda» (Dios es causa primera, pero hay causas segundas que operan con sus leyes), o el concepto de «necesidad condicionada» o ex-suppositione, opuesto al de ananke o "forzosidad». 19

La lucha contra lo fatídico e inamovible, contra la implacabilidad de la fisis, había generado un nuevo espacio en el que el hombre podía actuar, lícitamente, sobre la salud y sobre la enfermedad. Des de esta perspectiva, Laín

16. Para la anatomía de Mondito, cfr.: Loris PREMUdA, Storia della iconografia anatomica, Ciba Edizione, 1993, p. 52. Cfr. también, ID., "Anatomía en la Baja Edad Media», en Pedro Laín Entralgo (dir.), Historia universal de la medicina, cit., p. 300 y s.

17. Fragmento extraído de un trabajo de doctorado de Oriol FARRÉs JUSTE, estudiante de filosofía en la UAB: La pesta negra, una interpretació de la crisi epidèmica baixmedieval, p. 20 (inédito).

18. «Puede decirse que originariamente toda la radical innovación que el cristianismo introduce en filosofía es una nueva idea del mundo». Xavier ZUBIRI citado por Diego GrACIA y José Luis PESET, "La medicina en la Baja Edad Media Latina», en Pedro LAÍn ENTRALGO (dir.), cit., p. 344.

19. Citado por Daniel Gracia y José Luis PESET, «La medicina en la Baja Edad Media Latina», en Pedro Laín EnTRalgo (dir.), cit., p. 344. 
Entralgo nos recuerda que ciertamente «los remedios curan porque Dios quiere», pero también «por sus propiedades naturales» — y ahí residía la tarea del farmacólogo. ${ }^{20} \mathrm{El}$ hombre medieval se consideraba a sí mismo incapaz de gobernar aquello que en la creación es o sucede por «necesidad absoluta». Pero cuando la experiencia y la razón alcanzaban a conocer el mecanismo de aquello que sucede por "necesidad condicionada» podía ingeniar, mediante su ars, los recursos operativos para torcer en provecho propio el curso natural y espontáneo de los acontecimientos. Sólo entonces, conocedor de los límites de su finitud, podría convertirse en dueño de su propia naturaleza y sólo entonces podía, en consecuencia, aspirar a evadir o guarecer efectivamente la enfermedad.

La noción de una ars entendida como creación nace con Arnau de Vilanova y procede del voluntarismo antropológico y teológico de la Baja Edad Media. Su afirmación articuló, muy probablemente, el primer impulso de acercamiento de la medicina medieval a la línea de un horizonte científico-positivista todavía distante. En la definición de ars se vislumbraban, por un lado, el afán de novedad, el renovado interés por la realidad del mundo y por el valor epistemológico de las causas segundas que, más adelante, constituirían la condición de posibilidad para una ciencia experimental. Por otro lado, la reivindicación de la ars médica propició la emergencia de un atisbo de libertad propio del individualismo incipiente (Laín), tomando como trasfondo una realidad social que substituía, paulatinamente, la vida feudal por la vida burguesa. ${ }^{21}$ Sirva de paso, la transformación social señalada, para sugerir el estrecho vínculo existente entre revolución médica y revolución social como condición sine qua non de la primera: a diferencia de otras disciplinas como la ciencia física, la consolidación de una ciencia médica exige democracia y no jerarquía, puesto que el motor fundamental de su desarrollo es, precisamente, la elevación del concepto de salud como derecho elemental del ciudadano. En definitiva, la medicina medieval, si bien conjuntaba en su ars — con palabras de Vilanovael «experimentum» y la «ratio», requería todavía una culminación de las transformaciones sociales para consolidarse como disciplina moderna y autónoma. Mientras ésta no se produjera, las aportaciones medievales quedarían reducidas a una cristianización de la medicina griega — aceptada en sus patologías, aunque no en sus implicaciones ideológicas- a una cierta apertura técnica de los saberes y a un tímido movimiento del pensamiento hacia el libre albedrío. Del mismo modo que el médico hipocrático servía a la naturaleza, el médico escolástico se convirtió en servidor de la potencia ordenadora de Dios. Sin embargo, entre la concepción aristotélica de la vida como animación de la materia y el mecanicismo cartesiano ${ }^{22}$ que aplicaba a la máquina del organismo las

20. En lo que se refiere a la Ars Medica de Arnau de Vilanova, cfr. Pedro Laín Entralgo, La historia clínica, Barcelona: 1961, p. 68 y s.

21. Pedro Laín Entralgo (dir.), Historia universal de la medicina, cit., p. 352-354.

22. G. CANGuilhem, «Vie», en Encyclopedie universalis. Se confrontan, entre otros, los dos paradigmas fundamentales: la vida entendida como animación, propia del mundo antiguo y medieval, y la vida como mecanismo, que dará nacimiento a la ciencia biológica moderna. Sustitución del modelo aristotélico por el modelo cartesiano. 
leyes explicativas de la materia, mediaba un abismo todavía por resolver. Por ello creemos que durante el siglo XIV no hubo ruptura epistemológica en medicina, como tampoco la hubo en cosmología hasta Copérnico; en anatomía hasta Vesalio, o en física hasta Galileo. En la historia de la ciencia, quizá más que en cualquier otra disciplina, raras son las ocasiones en las que podemos acercarnos al mundo medieval con una visión desempañada de los logros posteriores cuya consecución cabalga a lomos de unas revoluciones, durante el siglo XIV, todavía por acontecer. 